\title{
Synthetic Scotophobin: Analysis of Behavioral Effects on Mice
}

\author{
DAVID H. MALIN ${ }^{2,3}$ \\ Department of Psychology and Mental Health Research Institute, University of Michigan \\ Ann Arbor, Michigan
}

(Received 21 December 1973)

\begin{abstract}
MALIN, D. H. Synthetic scotophobin: analysis of behavioral effects on mice. PHARMAC. BIOCHEM. BEHAV. 2(2) 147-153, 1974. - Scotophobin is a peptide previously identified [14] as the behaviorally active factor in brain extract of rats trained in passive dark avoidance. Mice injected intraperitoneally with a synthetic version of scotophobin or placebo were tested without reinforcement in a box with one dark and two white chambers. Scotophobin recipients spent less time than controls in the dark chamber. When strict precautions were taken against chemical degradation, the dose-response relationship remained stable for months and resembled that found by Ungar for natural scotophobin. A content analysis of all frequent mouse behaviors in the test apparatus delineated a more detailed scotophobin behavior pattern. Scotophobin elevated emotionality as measured by defecation rate when mice were locked in the dark box, but not when they were locked in a white or transparent box. The dark box as used in the original rat training situation produced the maximal drug induced avoidance effect. Avoidance was reduced by stimulus changes involving wall color, illumination, and the grid floor. Thus scotophobin induction of avoidance behavior as well as heightened emotionality appears to show stimulus specificity.
\end{abstract}

Scotophobin Passive avoidance Emotionality Stimulus specificity

SEVERAL laboratories $[4,6,7,15,16]$ have reported that injection of crude brain extract from rats shocked in a dark compartment can induce spontaneous avoidance of this compartment in recipient rats or mice. Ungar, Desiderio, and Parr [14] claim to have isolated, analyzed, and synthesized the active substance in one such brain extract. This substance, named scotophobin, is a peptide of fifteen amino acid residues. It is detectable by chemical means in the brains of trained rats or mice, but not in those of untrained animals. The ability of synthetic scotophobin to induce avoidance of dark compartments has been confirmed in fish [2,8,13] and in mice [9].

In the latter paper (a joint publication of two laboratories) this laboratory reported that mice injected with 1.5 , 2.2 , or $3.0 \mu \mathrm{g}$ of synthetic scotophobin spent significantly less time than placebo controls in a dark compartment when given a free choice for 3 min between light and dark compartments. However, in these previously reported experiments, this investigator, unlike Ungar, was unable to find any effect of synthetic scotophobin at a dose of $0.5-0.6 \mu \mathrm{g}$, the optimal dose for natural scotophobin from trained animals [14]. This failure was tentatively attributed to partial degradation of the synthetic scotophobin, which had been kept in methanol solution. Another possible explanation might be a non-equivalence between synthetic and natural scotophobin resulting from faulty chemical analysis of the latter as alleged by Stewart [12].

The first objective of the subsequent research reported here was to develop a way of handling and storing scotophobin that would prevent its progressive degradation and loss of biological effectiveness. It was hoped that this stabilization would make possible an exact replication - one obtained without multiplying the dose, as well as a meaningful series of dose-response experiments.

Aside from these quantitative considerations, there is the more basic qualitative question: is the biosynthesis of scotophobin an integral step in the acquisition and retention of a specific learned response - conditioned fear of a dark box? Scotophobin might lack specificity in two ways: in its origins or in its effects.

First, as Goldstein [5] has recently pointed out, the biosynthesis of this molecule might be caused by stimulation accompanying training (shock trauma, handling, etc.) rather than by any specific learning process. This possibility, currently under study by the author, will not be dealt with further in this paper.

A second possibility is that the response induced by scotophobin in naive recipients is only superficially related to conditioned fear of the dark box. Does scotophobin induce something like fear in recipients? And is such fear

\footnotetext{
${ }^{1}$ Most of the research reported here was performed at the University of Michigan; that part performed at Baylor College of Medicine was supported by National Institute of Education grant OEG-0-72-0699.

${ }^{2}$ Present address: Baylor College of Medicine, Houston, Texas, U.S.A.

${ }^{3}$ Thanks to James V. MoConnell for guidance and encouragement of this research.
} 
stimulus bound, that is, selectively elicited by the dark box? Or does scotophobin simply induce some general state-of-the-organism (unconditional fear, excitement, passivity, sensory deficit, etc.) which coincidentally reduces the animal's time spent in dark places.

A first, almost naturalistic, approach to these latter questions was to record all significantly altered behaviors during testing in addition to mere choice of compartment. Not only does this procedure present a more complete profile of the effects of scotophobin, but it may provide more sensitive indicators of the potency of the material than does dark-box time alone. Further experiments tested whether scotophobin affected a direct measure of fear or emotionality and whether any increase in emotionality was generalized or specific to the dark chamber. A final investigation was concerned with just what it was about the dark chamber that served as the stimulus for the avoidance response in injected animals.

\section{EXPERIMENT 1}

\section{Method}

Scotophobin, synthesized by Parr and Holzer [10] and generously donated by $\mathrm{G}$. Ungar, arrived as a powder under vacuum. New handling and storage procedures were employed to prevent contact with air and particularly with water vapor, which might hydrolyze the peptide bonds of scotophobin. The entire sample was dissolved in deionized distilled water, and the solution was divided into aliquot parts, each containing enough doses for one experiment. Thus aliquots of the same original sample were used for all tests to be reported. All portions were placed in VirTis vac-seal vials and were simultaneously lyophilized and then sealed under the lyophilizer vacuum. The vials were placed in a refrigerated vacuum dessicator on a layer of Drierite. The vials were removed one by one as needed for experiments and the contents were redissolved for injection.

Animals were 98 male, mature Swiss mice (Spartan Laboratories, Lansing, Michigan), 25 to $30 \mathrm{~g}$ in weight, housed individually in transparent plastic cages and maintained on a normal $12 \mathrm{~h}$ light cycle and ad lib food and water. These mice had been pre-screened for preference for the dark chamber of the test apparatus (at least $90 \mathrm{sec}$ spent in dark box during $3 \mathrm{~min}$ of free choice). About half of the mice screened had to be rejected. Each experimental group of 7 mice was injected I.P. with a given dose of scotophobin, always dissolved in $0.25 \mathrm{ml}$ of distilled water. Each control group consisted of 7 mice injected only with the same volume of distilled water. Both solutions and animals were coded and all testing was performed blind. Each animal was tested successively at $22,30,46$, and $78 \mathrm{hr}$ following injection.

The testing apparatus was a replica of that used in Ungar's laboratory [15]. The test animals were allowed to roam freely through 3 Plexiglas boxes, each $15 \mathrm{~cm}$ high, arranged in a row and connected by short corridors. At one end was a box $25 \mathrm{~cm}$ long and $15 \mathrm{~cm}$ wide with white Plexiglas walls and floor and a transparent top. In the center was a similar white box $30 \mathrm{~cm}$ long and $15 \mathrm{~cm}$ wide. At the other end was a smaller box, $20 \mathrm{~cm}$ long and $10 \mathrm{~cm}$ wide with opaque black Plexiglas walls and top. In this particular experiment, its grid floor was covered with black Plexiglas. Connecting passages were $7 \mathrm{~cm}$ in all dimensions. Each animal was initially placed in the center box and its score consisted of the number of seconds spent in the black box out of a 3 min test period, as recorded by a blind observer with stopwatch.

Because of animal housing limitations, dose-response testing was done in several successive experiments. In each such experiment there was a control group of 7 mice injected I.P. with $0.25 \mathrm{ml}$ distilled water. In the first experiment three experimental groups receiving 3.0, 2.0, and $1.0 \mu \mathrm{g}$ were tested along with a control group. A second experiment tested $0.67,0.4$, and $0.25 \mu \mathrm{g}$ along with controls. To test the reliability of these results and the stability of the material, a repeated test was made of $0.67 \mu \mathrm{g}$ and controls, as well as an interpolated dose of $0.5 \mu \mathrm{g}$, over 3 months after the second experiment.

\section{Results}

Table 1 shows the dark box time (mean and standard deviation) on each individual test as well as the overall average of all 4 tests. The averages of the 3 control groups were quite similar $(117,128$, and 127). When the Kruskal-Wallis analysis of variance by ranks was performed on the 3 control groups, the resulting $\mathrm{H}$ statistic was far from significant $(\mathrm{H}=0.25, p<0.8)$. Thus it appears that different dates or conditions of testing did not in themselves alter dark preference. When a similar analysis was performed on all 7 groups receiving different doses of scotophobin, the result was highly significant $(\mathrm{H}=24.3, p<0.01)$, demonstrating that dark box time is dependent on the dosage of scotophobin. It is of particular interest that the two groups receiving $0.67 \mu \mathrm{g}$ in different experiments about 3 months apart performed almost identically. The Mann-Whitney U statistic for their ranked scores did not approach significance $(U=21)$. This suggests a good degree of retest reliability, as well as the successful stabilization of the chemical material for a fairly prolonged period.

The bottom rows of Table 1 express the results as a ratio of the average dark box time (over all four tests) of each dosage group to the average dark box time of its respective control group. Scotophobin produced a strong positive effect relative to controls only within a narrow dose range encompassing $0.4,0.5$, and $0.67 \mu \mathrm{g}$. (The corresponding $p$ values according to the Mann-Whitney $U$ Test were $\leqslant 0.02$, 0.05 , and 0.02 .) This is very close to the optimum dose range $(0.5-0.6 \mu \mathrm{g})$ for natural brain scotophobin [14]. Doses of $0.25,1.0$ and $2.0 \mu \mathrm{g}$ produced small, nonsignificant effects. The largest dose, $3.0 \mu \mathrm{g}$, appeared to cause an inverse effect - scotophobin mice spent significantly more time in the dark box than controls ( $U=10, p \leqslant 0.05)$. Ungar (personal communication) has also noticed an inversion of behavioral effects in this general range of dosage. The significance of this reversal is unknown, but similar dose-related inversions have been reported with natural brain extracts from trained animals [11]. The maximal effects in this experiment were somewhat less than those reported earlier from our laboratory [9]. A grid floor was used in the earlier experiments, and the results of Experiment 3 suggest that this might explain the difference.

The onset of the effect was rather gradual since maximal differences between scotophobin recipients and controls were usually not observed until 30 or $46 \mathrm{hr}$ postinjection. Subsequent experiments by this author and colleagues (Malin and Radcliffe, in preparation) show that the size of the effect is primarily a function of time postinjection rather than number of exposures to the test situation. This might reflect time required for distribution of scotophobin 
TABLE 1

EFFECT OF DIFFERENT DOSES OF SCOTOPHOBIN ON DARK BOX TIME (DBT)

(EACH GROUP, $\mathrm{N}=7$ )

\begin{tabular}{|c|c|c|c|c|c|c|c|c|c|}
\hline \multicolumn{10}{|c|}{ DBT \pm S.D. } \\
\hline Expt 1a & Test at & \multicolumn{2}{|c|}{ Control } & \multicolumn{2}{|c|}{$\mathrm{SP} 3 \mu \mathrm{g}$} & \multicolumn{2}{|c|}{$2 \mu \mathrm{g}$} & \multicolumn{2}{|c|}{$1 \mu \mathrm{g}$} \\
\hline \multirow[t]{6}{*}{ MARCH } & $22 \mathrm{hr}$ & 137 & 32 & 165 & 13 & 120 & 47 & 159 & 16 \\
\hline & $30 \mathrm{hr}$ & 113 & 39 & 143 & 22 & 134 & 43 & 95 & 63 \\
\hline & $46 \mathrm{hr}$ & 119 & 41 & 160 & 19 & 99 & 61 & 104 & 57 \\
\hline & $78 \mathrm{hr}$ & 100 & 60 & 140 & 24 & 110 & 58 & 91 & 54 \\
\hline & Mean & 117 & 28 & 156 & 27 & 116 & 39 & 112 & 41 \\
\hline & $\%$ of controls & & & \multicolumn{2}{|c|}{$133 \%$} & \multicolumn{2}{|c|}{$99 \%$} & \multicolumn{2}{|c|}{$95 \%$} \\
\hline Expt $1 b$ & Test at & \multicolumn{2}{|c|}{ Control } & \multicolumn{2}{|c|}{ SP $0.67 \mu \mathrm{g}$} & \multicolumn{2}{|c|}{$0.4 \mu \mathrm{g}$} & \multicolumn{2}{|c|}{$0.25 \mu \mathrm{g}$} \\
\hline \multirow[t]{6}{*}{ APRIL } & $22 \mathrm{hr}$ & 128 & 51 & 102 & 72 & 105 & 57 & 137 & 46 \\
\hline & $30 \mathrm{hr}$ & 119 & 37 & 79 & 55 & 78 & 62 & 112 & 51 \\
\hline & $46 \mathrm{hr}$ & 131 & 64 & 76 & 59 & 82 & 68 & 98 & 67 \\
\hline & $78 \mathrm{hr}$ & 136 & 50 & 81 & 61 & 76 & 58 & 103 & 64 \\
\hline & Mean & 128 & 30 & 84 & 48 & 85 & 52 & 113 & 50 \\
\hline & $\%$ of controls & & & \multicolumn{2}{|c|}{$65 \%$} & \multicolumn{2}{|c|}{$66 \%$} & \multicolumn{2}{|c|}{$88 \%$} \\
\hline Expt 1c & Test at & \multicolumn{2}{|c|}{ Control } & \multicolumn{2}{|c|}{$\mathrm{SP} 0.67 \mu \mathrm{g}$} & \multicolumn{2}{|c|}{$0.5 \mu \mathrm{g}$} & & \\
\hline \multirow[t]{6}{*}{ JULY } & $22 \mathrm{hr}$ & 138 & 21 & 103 & 63 & 90 & 59 & & \\
\hline & $30 \mathrm{hr}$ & 104 & 57 & 81 & 72 & 61 & 43 & & \\
\hline & $48 \mathrm{br}$ & 124 & 61 & 68 & 50 & 111 & 69 & & \\
\hline & $78 \mathrm{hr}$ & 143 & 23 & 70 & 54 & 92 & 67 & & \\
\hline & Mean & 127 & 24 & 80 & 43 & 88 & 38 & & \\
\hline & $\%$ of controls & & & \multicolumn{2}{|c|}{$63 \%$} & \multicolumn{2}{|c|}{$69 \%$} & & \\
\hline
\end{tabular}

to highly localized receptors in brain or necessary to complete a chemical or structural change triggered by scotophobin.

\section{EXPERIMENT 2}

Experiments where only dark box time is recorded might leave undetected multiple behavioral effects of scotophobin unrelated to dark box time. Therefore, a naturalistic, but systematic, approach was used to define and then test the general behavior syndrome induced by scotophobin in the black-white apparatus. As a pilot procedure, during the previously published experiments with scotophobin, the author simply tried to note all the reasonably frequent behaviors observable during testing of scotophobin and control mice under blind conditions. A code letter and a scoring criterion were assigned to each of these behaviors.
After the data from the experimental and control animals were decoded, five of these behaviors appeared to differentiate the two groups. The first behavior is called turning away. The animal approaches within a body length of the black doorway with its head pointed toward the door and then turns and walks in the other direction. The second response pattern is that of hesitation in the doorway. The animal crouches on the edge of the door into the black compartment with its body at an oblique angle to the threshold and alternately looks into the black box and then into the white chamber. This is a particularly unusual and striking behavior. The third response is sitting in the tar white corner. The animal settles down in one of the corners furthest away from the entrance to the black chamber for at least five seconds. This is often accompanied by the fourth behavior, grooming for $5 \mathrm{sec}$ or more. The fifth behavior is dropping at least three fecal boluses in the test 
apparatus. Experiment 2 reexamined the effect of scotophobin on these five preselected measures, thus validating them beyond post-hoc analysis.

\section{Method}

Animals were 14 pre-scrcened mice injected with $0.67 \mu \mathrm{g}$ of scotophobin and 7 animals injected with distilled water. Each mouse was tested blind in five $3 \mathrm{~min}$ sessions in the black-white box, occurring at $20,24,44,48$, and $72 \mathrm{hr}$ post-injection. Each occurrence of the behaviors described above was noted along with the dark box time of each mouse. Otherwise, all details of the method were the same as in Experiment 1.

\section{Results and Discussion}

Figure 1 indicates the average behavior counts for each group over the entire experiment. As the figure shows, the scotophobin group ranked significantly higher than the controls on each individual measure. A composite measure was also obtaincd by simply adding the counts on all five behaviors for each mouse. The scotophobin group averaged over four times higher on this composite measure and the two sets of composite scores were significantly different at the 0.001 level. In contrast, the overall dark-box scores for these two groups were only significantly different at the 0.05 level. Thus, the composite behavior score may be at least as sensitive an indicator of the scotophobin effect and

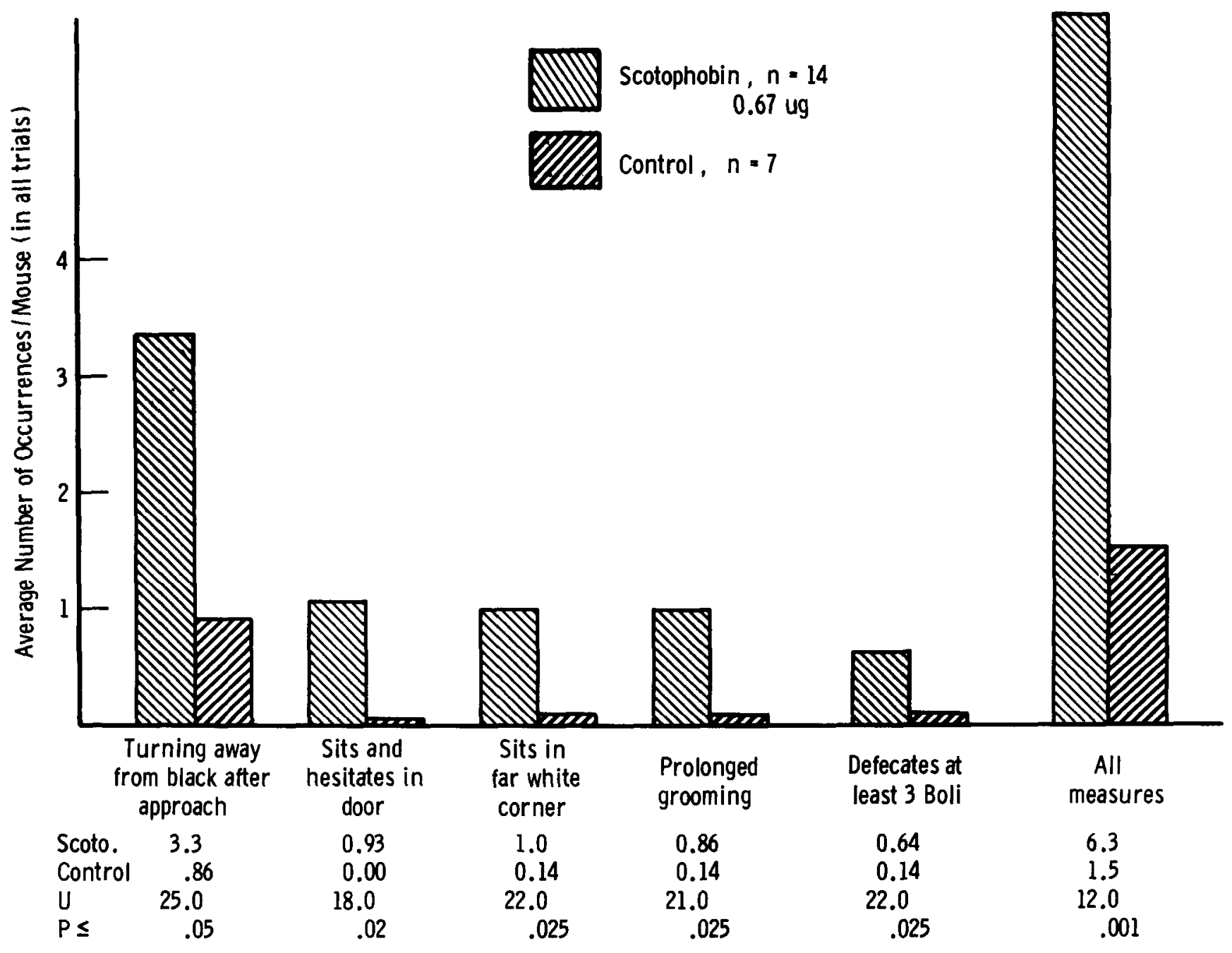

FIG. 1. Effect of scotophobin on various behavioral measures. 
might well be used to supplement the measurement of dark-box time.

By reviewing the raw data one can arrive at a very rough description of a typical sequence of scotophobin induced behaviors. The first sign of the effect in any given mouse is the appearance of turning away behavior, generally on the first day. This becomes more frequent and may intensify into prolonged hesitation at the doorway. At the apparent peak of the effect, when dark box time falls to near zero on the second or third day, the mouse retreats to the far corner of the white compartment, where he sits and grooms himself and no longer even approaches the black box very closely. Most control animals continue throughout to spend a moderate amount of time in the white compartment. However, this time is spent in rapid exploration without settling down, and the dark chamber is re-entered without hesitation. In general, then, this more detailed description of scotophobin's behavioral effect is not inconsistent with the idea that this substance makes mice a little uneasy about the dark compartment.

\section{EXPERIMENT 3}

Can one necessarily conclude from the fact that scotophobin recipients spend less time in the dark box that the injection induces fear of the dark? One problem is that no fear has been directly measured or demonstrated but merely inferred from a change in preference. Furthermore, if the injection can actually induce fear, is it, like conditioned fear, selectively triggered by exposure to the dark compartment? Or is the animal simply afraid to begin with and consequently less ready to enter and explore dark places?

The question, then, is whether scotophobin has no effect, a generalized effect, or a stimulus-specific effect on the emotionality of mice. To approach this question, one first needs a direct measure of emotionality. One of the oldest, simplest, and perhaps most reliable measure is defecation rate - the number of fecal boluses dropped by an animal during a given time period.

\section{Method}

Thirty mice, after having been screened for dark preference, were habituated to the black-white apparatus to reduce the baseline emotional response by reducing the novelty of the environment. They were allowed to roam freely through the apparatus on 3 successive days for $3 \mathrm{~min}$ each day. About an hour after the third habituation session, 14 of these mice were then injected with $0.5 \mu \mathrm{g}$ of scotophobin in $0.25 \mathrm{ml}$ distilled water, while 16 mice received only the distilled water. Forty-eight hr postinjection, each of 7 experimental and 8 control animals was forced through the doorway of the smaller white chamber (at the opposite end from the black box) and was locked in that compartment. Three min later the animal was removed and the number of fecal droppings left behind was recorded. Twelve hr later the same procedure was followed using the black chamber (with the grid floor exposed). To control for possible confounding effects of time or sequence, the experiment was performed in counterbalanced order with the other 15 mice. These were tested at $48 \mathrm{hr}$ post-injection in the black chamber and at $60 \mathrm{hr}$ in the white chamber. At $72 \mathrm{hr}$ post-injection all mice were placed into an entirely different and novel situation, a transparent Plexiglas cube
(20 $\mathrm{cm}$ to each side) with grid floor. Again, the number of boluses left behind after 3 min was recorded.

\section{Results}

The results of this experiment are shown in Fig. 2. Compared with their controls, scotophobin animals showed less apparent emotionality in the white chamber and more in the dark chamber. A difference score (boluses in black minus boluses in white) was obtained for each mouse. When the scores of the two groups were ranked logether, the resulting Mann-Whitney U statistic was highly significant $(\mathrm{U}=12, p \leqslant 0.001)$. This finding indicates a significant, non-parametric interaction effect [1] of drug and environment in determining emotionality. In other words, the direction of scotophobin's effect on emotionality depended significantly on the testing environment.

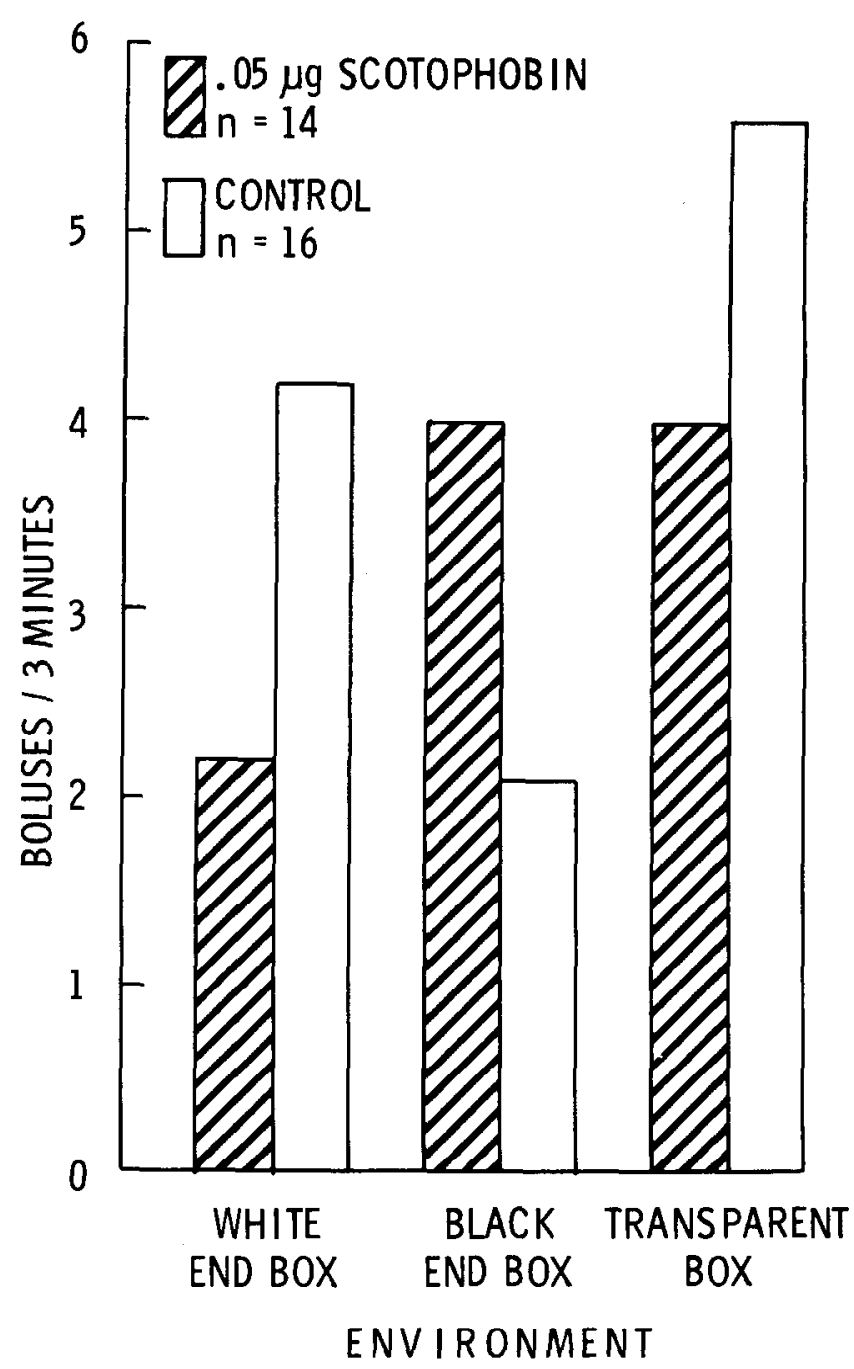

FIG. 2. Interactive effect of scotophobin and envirunment on emotionality. 
Since neither group had been habituated to the transparent box, the scores for this chamber tended to be the highest of all. However, the control animals scored even higher than the scotophobin mice. (The difference was not significant.) Thus, there is no evidence that scotophobin raises emotionality in a novel, neural environment.

Further experiments of this sort are planned to test other doses of scotophobin, particularly doses having a minimal or reversal effect on dark box time.

\section{EXPERIMENT 4}

Experiment 3 showed that the dark box selectively aroused heightened emotionality as well as behavioral avoidance in scotophobin-treated mice. There remains the question of just what stimulus components of this dark compartment trigger these effects. There are, after all, a number of differences between the dark and light compartments of Ungar's apparatus. The dark box differs from the light in having black walls, an opaque top, very low illumination, a grid floor, and smaller size. Which of these is the stimulus that triggers aversion?

One approach to this general problem is to begin with the intacl testing appartus and remove various features of the dark box, while observing what effect these subractions have on the magnitude of scotophobin-induced aversion.

\section{Method}

Four different configurations of the end box were employed: (1) the intact dark box as originally used by Ungar for the dark avoidance training of rats that induced the synthesis of natural scotophobin in their brains, (2) the dark box with the grid covered with black Plexiglas floor, (3) the dark box with covered floor and a transparent top, keeping the box at nearly normal room illumination, and (4) a white box with grid floor and transparent roof. In each case the end box was lined up with the two unaltered white boxes and test animals were allowed to wander at will through all three chambers for $3 \mathrm{~min}$. Four groups of 16 mice (all pre-screened for dark preference) were tested, one in each configuration. Half the animals in each group re- ceived $0.67 \mu \mathrm{g}$ scotophobin in distilled water and half received water alone. Mice were tested blind at 24 and $48 \mathrm{hr}$ post-injection. Mice in Condition (4) were also scored for activity level at $48 \mathrm{hr}$. This was done by counting the total number of crossings from any of the 3 white boxes to another. Other details of the methods are the same as in Experiment 1 .

\section{Results and Discussion}

As in Experiment 1, there were only small differences between the various groups at $24 \mathrm{hr}$. At $48 \mathrm{hr}$, however, these intensified into a clear trend as shown in Table 2. Analysis of variance revealed a significant main effect of scotophobin administration $(p<0.01)$ and a significant interaction of this variable with end box configuration $(p<0.05)$, even though there was no significant main effect of end box configuration per se. The avoidance effect of scotophobin thus depended significantly on the properties of the end box. When each scotophobin group is compared with its respective controls, the results may be simply summarized: the closer the resemblance between the test situation and the original donor training situation which Ungar used to induce natural scotophobin, the greater the size and statistical significance of the scotophobin effect. The scotophobin-treated mice seemed to respond in some degree to an entire complex of stimuli associated with the dark box. To obtain a maximal avoidance effect this stimulus complex must be intact. While stimulus specificity was not absolute, stimulus changes produce a sort of generalization decreement resembling that found in testing normal memory. In short, the scotophobin effect is apparently stimulus or situation specific and this specificity may be finer and more complicated than was first imagined. It is interesting to note that the scotophobin effect disappears entirely when a white version of the normally dark end box is used. This tends to rule out such "non-specific" explanations of scotophobin reduced dark box time as position habit, altered preference for loose or close quarters, and increased passivity or decreased exploratory activity, as suggested by Frank et al. [3]. The failure of scotophobin to

TABLE 2

EFFECT OF CHANGES IN TEST APPARATUS. AVERAGE END BOX TIME IN SEC AS AFFECTED BY $0.67 \mu \mathrm{g}$ SCOTOPHOBIN AND END BOX CONFIGURATION (48 HR POSTINJECTION; EACH GROUP, $\mathrm{N}=8$ )

\begin{tabular}{|c|c|c|c|c|c|}
\hline End Box & Controls & Scotophobin & $\begin{array}{l}\text { Scotophobin/ } \\
\text { Control }\end{array}$ & $\mathrm{U}$ & $p \leqslant$ \\
\hline 1. Dark box intact & 123 & 48 & 0.39 & 2 & 0.001 \\
\hline 2. Dark box minus grid & 120 & 72 & 0.60 & 9 & 0.01 \\
\hline $\begin{array}{l}\text { 3. Dark box minus grid, } \\
\text { minus opaque top }\end{array}$ & 111 & 83 & 0.81 & 13 & 0.05 \\
\hline $\begin{array}{l}\text { 4. White box with grid, } \\
\text { minus opaque top }\end{array}$ & 66 & 76 & 1.13 & 26 & NS \\
\hline Inter-box crossings & 8.13 & 7.13 & 0.88 & 28 & NS \\
\hline
\end{tabular}


affect the number of crossings in Condition (4) (no color contrast) also tends to rule out the latter possibility.

Incidentally, a comparison of Conditions (1) and (2) tends to explain the rather shallow effects in Experiment 1, which was conducted without the grid floor, as in Condition (2).

\section{CONCLUSIONS}

With appropriate precautions against instability, the behavioral effect of synthetic scotophobin can be reliably obtained. Furthermore, the optimum dose range is close to that of natural scotophobin isolated from rat brain [14]. When the testing conditions were comparable (Condition (1), Experiment 3), the degree of effect at the optimal dose resemeled that previously reported for natural scotophobin (mean DBT of 48 and $40 \mathrm{sec}$ respectively). This strongly suggests that synthetic and natural scotophobin are substantially similar, despite Stewart's [12] objections, particularly since synthetic scotophobin with only one modified amino acid residue [14] may lose over $90 \%$ of its activity (its optimum dosc increasing tenfold).

These dose-response results with pure synthetic material may shed some light on problems of replicability in chemical transfer experiments with crude natural brain extracts.
The effective dose range of the pure active material is so narrow that the effect of a given dose of the corresponding natural extract would depend sharply on (1) the amount of this substance synthesized per donor brain as determined by the details of donor training and maintenance, (2) the yield of the extraction process, $(3)$ the stability of the molecule as affected by handling and storage procedures.

The outcome of Experiments 2, 3, and 4 is consistent with the hypothesis that scotophobin induces uneasiness in environments resembling those in which the original donor animals were punished. This does not mean, of course, that all conceivable non-specific interpretations have been eliminated. For example, an animal with a raised visual threshold might become uneasy in a dark environment. However, subsequent experiments by this author and colleagues (Malin and Radcliffe, in preparation) suggest that scotophobin in mice does not lower visual sensitivity in dark environment. Scotophobin recipients required no higher levels of illumination than did controls to maintain accuracy in a visually guided escape task. Much further analysis is needed, but the data thus far are consistent with the hypothesis that the formation of this peptide substance could be one component step in the acquisition of a particular learned response.

\section{REFERENCES}

1. Bradley, J. V. Distribution-Free Statistical Tests. Englewood, N. J.: Prentice-Hall, 1968, pp. 138-141.

2. Bryant, R. C., N. N. Santos and W. L. Byrne. Synthetic scotophobin in goldfish. Science 177: 635-636, 1972.

3. Frank, B., D. G. Stein and J. Rosen. Interanimal "memory" transfer: results from brain and liver homogenate. Science 169: $399-402,1970$.

4. Gay, R. and A. Raphelson. Transfer of learning by injection of brain RNA. Psychon. Sci. 11: 1-2, 1968.

5. Goldstein, A. Comments on "Isolation, identification and synthesis of a specific-behavior-inducing-brain peptide." Nature 242: $60-62,1973$.

6. Golub, A. M., L. Epstein and J. V. McConnell. The effects of peptides, RNA, and whole brain homogenates on avoidance behavior. J. Biol. Psychol. 11: 44-49, 1969.

7. Guttman, H. N. and L. Gronke. Passive transfer of learned dark and step-down avoidance. Psychon. Sci. 24: 107-109, 1971

8. Guttman, H. N., G. Matwyshyn and G. H. Warriner. Synthetic scotophobin-mediated passive transfer of dark avoidance. Nature 235: 26-27, 1972.

9. Malin, D. H. and H. N. Guttman. Synthetic rat scotophobin induces dark avoidance in mice. Science 178: 1220-1221, 1972.
10. Parr, W. and G. Holzer. The structure of the "memory-codeword" scotophobin. In: Memory and Transfer of Information, edited by H. P. Zippel. New York: Plenum Press, 1973, pp. 303-316.

11. Rosenblatt, F., J. T. Farrow and S. Rhine. The transfer of learned behavior by means of brain extracts. I and II. Proc. natn. Acad. Sci. U.S.A. 55: 548-555 and 787-792, 1966.

12. Stewart, W. W. Comments on the chemistry of scotophobin. Nature 238: 202-209, 1972.

13. Thines, G., F. Domagk and E. Schonne. The effect of synthetic scotophobin on the light tolerance of teleosts (Carassius auratus and Tinca tinca). In: Memory and Transfer of Information, edited by H. P. Zippel. New York: Plenum Press, 1973, pp. 363-371.

14. Ungar, G., D. M. Desiderio and W. Parr. Isolation, identification, and synthesis of a specific-behavior-inducing brain peptide. Nature 238: 198-202, 1972.

15. Ungar, G., L. Galvan and R. H. Clark. Chemical transfer of learned fear. Nature 217: 1259-1261, 1968.

16. Wolthuis, $O$. L. Inter-animal information transfer by brain extracts. Arch. int. Pharmacodyn. Ther. 182: 439-442, 1969. 